\title{
Malignant Mixed Tumor, Not Otherwise Specified
}

National Cancer Institute

\section{Source}

National Cancer Institute. Malignant Mixed Tumor, Not Otherwise Specified. NCI

Thesaurus. Code C121787.

A malignant myoepithelioma characterized by the presence of a minor ductal component. 